\title{
Predictability matters: role of the hippocampus and prefrontal cortex in disambiguation of overlapping sequences
}

\author{
Justine E. Cohen, ${ }^{1,2}$ Robert S. Ross, ${ }^{3}$ and Chantal E. Stern ${ }^{1,2}$ \\ ${ }^{1}$ Center for Memory and Brain, Rajen Kilachand Center for Integrated Life Sciences and Engineering, Boston University, Boston, \\ Massachusetts 02215, USA; ${ }^{2}$ Athinoula A. Martinos Center for Biomedical Imaging, Massachusetts General Hospital, Charlestown, \\ Massachusetts 02129, USA; ${ }^{3}$ Sychology Department, University of New Hampshire, Durham, New Hampshire 03824, USA
}

\begin{abstract}
Previous research has demonstrated that areas in the medial temporal lobe and prefrontal cortex (PFC) show increased activation during retrieval of overlapping sequences. In this study, we designed a task in which degree of overlap varied between conditions in order to parse out the contributions of hippocampal and prefrontal subregions as overlap between associations increased. In the task, participants learned sequential associations consisting of a picture frame, a face within the picture frame, and an outdoor scene. The control condition consisted of a single frame-face-scene sequence. In the low overlap condition, each frame was paired with two faces and two scenes. In the high overlap condition, each frame was paired with four faces and four scenes. In all conditions the correct scene was chosen among four possible scenes and was dependent on the frame and face that preceded the choice point. One day after training, participants were tested on the retrieval of learned sequences during fMRI scanning. Results showed that the middle and posterior hippocampus $(\mathrm{HC})$ was active at times when participants acquired information that increased predictability of the correct response in the overlapping sequences. Activation of dorsolateral PFC occurred at time points when the participant was able to ascertain which set of sequences the correct response belonged to. The ventrolateral PFC was active when inhibition was required, either of irrelevant stimuli or incorrect responses. These results indicate that areas of lateral PFC work in concert with the $\mathrm{HC}$ to disambiguate between overlapping sequences and that sequence predictability is key to when specific brain regions become active.
\end{abstract}

\begin{abstract}
We often rely on contextual information to retrieve specific events or sequences of events. Accumulating evidence indicates that interactions between the hippocampus (HC) and prefrontal cortex (PFC) are critical to the retrieval of events with overlapping elements that rely on contextual information (Rich and Shapiro 2009; Brown et al 2010, 2012). While some items or places have only one or two associations connected to them, other items may have multiple overlapping associations. For example, Chicago's O'Hare airport may have two distinct events connected to it - the time you were forced to stay in the airport overnight due to a snowstorm and when you got food poisoning from the sushi restaurant in the food court-whereas the local hospital has many associations-it is where you gave birth to each of your children and had a broken leg and visited your dad when he was sick. Is there a difference in how we retrieve memories that have few versus multiple overlapping associations? In this study we were interested in investigating the following questions: As relational load increases how does activation of the HC and PFC and their associated networks change? How does ability to predict or think through an upcoming sequence affect neural activation at both regional and whole brain levels? Disambiguating between similar events or associations is thought to rely on pattern separation, a process dependent on the dentate gyrus of the $\mathrm{HC}$ and related medial temporal lobe structures. Pattern separation involves the creation of distinct neural representations for stimuli that are the same but used in different contexts (Yassa et al. 2011; Yassa and Stark 2011; Reagh and
\end{abstract}

\section{Corresponding author: chantal@bu.edu}

Article is online at http://www.learnmem.org/cgi/doi/10.1101//m.047175. 117.
Yassa 2014; Bennett and Stark 2016). A series of studies in rodents have shown that the HC forms distinct representations for overlapping sequences in both spatial and nonspatial tasks (Frank et al. 2000; Wood et al. 2000; Agster et al. 2002). Human neuroimaging studies have also shown hippocampal activation during the encoding and retrieval phases of both spatial and nonspatial contextdependent memory tasks with overlapping stimuli, including single items and sequences (Bakker et al. 2008; Ross et al. 2009; Brown et al. 2010; Lacy et al. 2011; Newmark et al. 2013). Recently, multivoxel pattern analyses of functional magnetic resonance imaging (fMRI) in the HC has shown that learning causes differentiation of hippocampal activation patterns for overlapping stimuli (LaRocque et al. 2013; Favila et al. 2016; Tompary and Davachi 2017). In addition to these functional differences, the ability to accurately and efficiently disambiguate between overlapping mazes is correlated with structural differences in the $\mathrm{HC}$ and rostral dorsolateral PFC (Brown et al. 2014).

When items and associations are shared between sequences of events, the PFC is involved in synthesizing episodic memories into relational sets that evolve with experience and cognitive demands and contribute to behavioral flexibility (Miller and Cohen 2001; Eichenbaum 2004; Hasselmo 2005; Rich and Shapiro 2009). It is well established that the PFC plays an integral role in the retrieval of episodic memory, with different regions responding to different

2018 Cohen et al. This article is distributed exclusively by Cold Spring Harbor Laboratory Press for the first 12 months after the full-issue publication date (see http://learnmem.cshlp.org/site/misc/terms.xhtml). After 12 months, it is available under a Creative Commons License (Attribution-NonCommercial 4.0 International), as described at http://creativecommons.org/licenses/by-nc/ $4.0 \%$. 
cognitive and behavioral demands. Neuroimaging studies suggest that the dIPFC is active while maintaining, monitoring, and manipulating information in working memory (Petrides 1995, 2000; D’Esposito et al. 1999; Postle et al. 1999; Stern et al. 2000, 2001; for review see Nee et al. 2013), the vlPFC is involved in interference resolution (Badre and Wagner 2005; Jonides and Nee 2006; Caplan et al. 2007; Öztekin et al. 2009; Schlichting and Preston 2015), and the left vlPFC is active during the retrieval of contextual details of memory (Kahn et al. 2004; Badre and Wagner 2007; Chapados and Petrides 2015). Petrides (1994) proposed that the mid-vlPFC is involved in comparing and selecting stimuli held in short- and long-term memory and that this region is particularly important in situations that require disambiguation of information from memory (Petrides 2002). In addition, the left vlPFC has been involved in proactive interference when there are overlapping or similar associations. The vlPFC is involved both in inhibiting irrelevant, competing information and in selecting relevant information through connectivity with the HC (Nee et al. 2007; Oren et al. 2017). In addition to its role in working memory, the dIPFC is part of the cognitive control network and has been proposed to modulate attention through top-down executive control (Rowe et al. 2000; Miller and Cohen 2001). During retrieval of overlapping spatial routes (mazes), both the rostral lateral prefrontal and orbitofrontal cortices were recruited (Brown et al. 2010, 2012). These regions were significantly less active during the retrieval of nonoverlapping mazes.

Our goal was to examine the effects that varying degrees of relational overlap have on activity in MTL and PFC regions. Our task consisted of sequential associations made up of a picture frame (of varying colors, textures, and patterns) followed by a black and white photograph of a face within the picture frame followed by color photographs of four outdoor scenes (see Fig. 1). One of the four outdoor scenes correctly completed the sequence and the correct scene was dependent on which face and frame were initially presented. The control sequence had no overlap, the low overlap sequence overlapped with one other sequence, and the high overlap sequence overlapped with three other sequences. In addition, the task was divided into three time points that enabled us to examine how the ability to access the possible upcoming stimuli or predict the correct response affected neural activation at both regional and whole brain levels. This task differed from previous tasks examining disambiguation in that it involved a sequence of nonspatial stimuli and multiple degrees of overlap between associations across conditions (Brown et al. 2010; Ross et al. 2011). Increasing the number of relational associations connected to one stimulus can result in increased interference and cognitive demand and decreased ability to look ahead in a sequence completion task.

Based on previous studies of hippocampal function, we expected to find increased hippocampal activation in this task. In both animals and humans, memory for sequences of items has been shown to rely on the HC (Agster et al. 2002; Schendan et al. 2003; Brown et al. 2010, 2014; Hsieh and Ranganath 2015), and previous work in humans has suggested that different functions may be related to detailed (posterior) and gist-like (anterior) hippocampal representations (Poppenk and Moscovitch 2011; Nadel et al. 2013; Poppenk et al 2013). In our task, the low overlap condition contained a larger pool of stimuli than the high overlap condition. In contrast, the high overlap condition did not contain as many stimuli as the low overlap condition but there was an increased demand to separate, or disambiguate, each trial's sequence from the three sequences with which it overlapped. Therefore, we expected to find increased activation in both the anterior and posterior $\mathrm{HC}$ where item and context information are combined and the anterior $\mathrm{HC}$ which is thought to relay context information to the PFC (Eichenbaum et al. 2012; Komorowski et al. 2013; Poppenk et al. 2013). We also expected to see increased activation in the anterior $\mathrm{HC}$ during the association period of the low overlap condition because it is at this point that the $\mathrm{HC}$ was able to relay information to the PFC pointing to the appropriate response to complete the sequence.

We expected to find differential activation in the vlPFC, based on differences in relational load and the degree of interference from overlapping items (Jonides and Nee 2007; Nee et al. 2006, Oren et al. 2017). Because the high overlap condition had the highest degree of interference from items not relevant to the current trial, we expected to see the most vlPFC activity during this condition, particularly at the association time point. In addition we anticipated differences in activation in the dIPFC between conditions. One proposed role of the dlPFC is executive control of neural regions processing task relevant representations involved in response preparation (Rowe et al. 2000). In our task, we would expect the dIPFC to be active when there is information present that determines the representations relevant to the trial. In the low overlap condition, this would be during both the cue and association periods and in the high overlap condition this would be during the association period. In the control condition, the participant should be able to predict the entire sequence at the cue period. However, due to the simplicity of the sequence used in the control condition and the number of times this trial is repeated, we did not expect to see activation in the PFC.

\section{Results}

\section{Behavioral data}

Participants were trained on the task $1 \mathrm{~d}$ prior to performing the task during fMRI scanning. The task consisted of one of 13 possible picture frames, each differentiated by color, pattern, and/or texture, presented on the computer monitor followed by the photograph of a face within the picture frame, followed by four outdoor scenes (see Fig. 1). The correct response changed with each frame/ face combination that was presented.

The control consisted of a simple frame-face-scene sequence. The low overlap condition consisted of four pairs of sequences. Each frame was paired with two faces and two scenes. For example, when the green frame was shown before the old man the correct response was the vineyard but when paired with the younger man the correct response was the grassy field. However, when the old man was shown after the fire frame the correct
Figure 1. Participants learned sequences of contextual cues and associated face-scene combinations The same faces and scenes were presented with multiple frames and correct responses were dependent on the frame initially shown. 


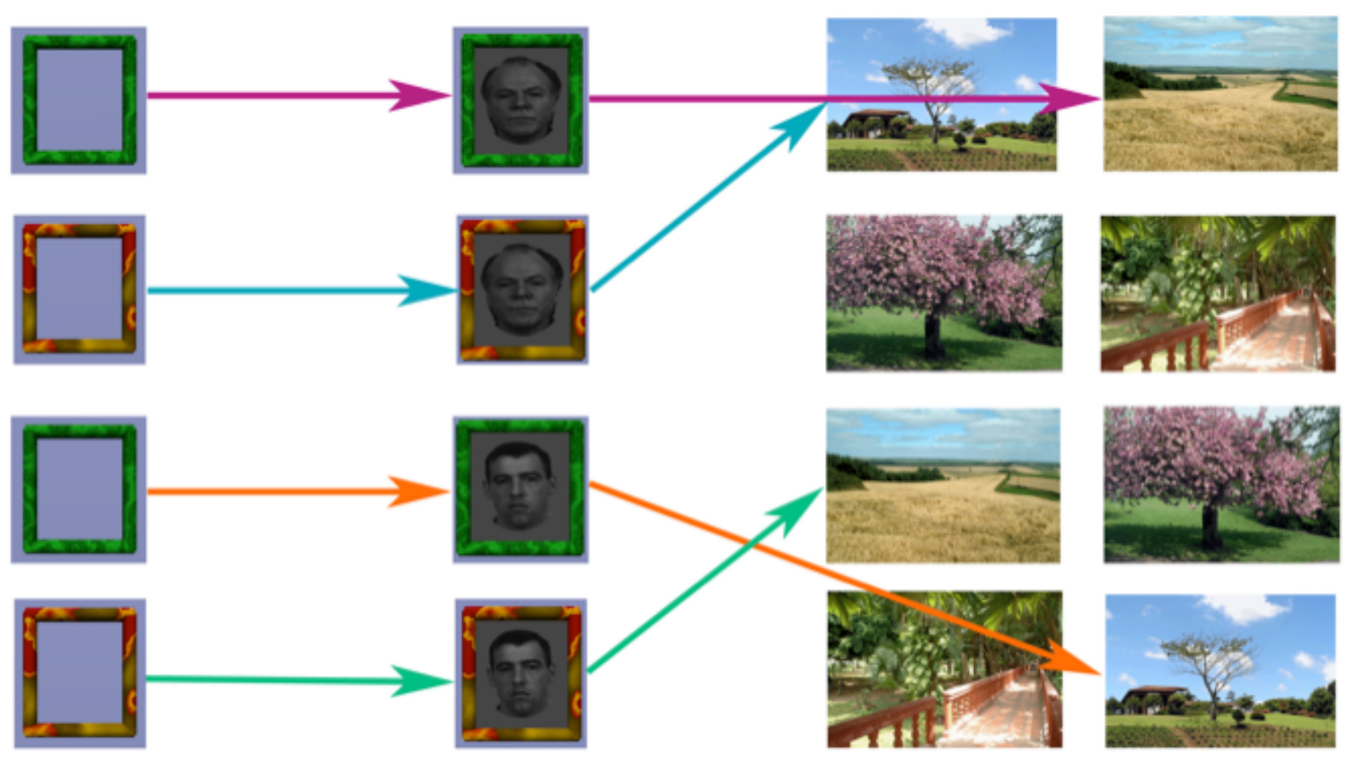

Figure 2. Low overlap condition. Each frame and face was associated with two scenes, and the correct response was dependent on the frame initially presented. This condition included four sets of two frames, two faces, and two scenes. With each successive sequence element, participants were better able to predict the correct response in this condition.

response was the grassy field and when the young man was shown after the fire frame the correct response was the vineyard (see Fig. 2).

The high overlap condition had the highest degree of overlap between associations and the lowest level of predictability. In the high overlap condition, the participant learned that each frame was associated with four different faces and four scenes. There were four frames in the high overlap condition and a total of sixteen overlapping associations (see Fig. 3).
We examined differences in accuracy and reaction times between the control, low overlap, and high overlap conditions while participants performed the task in the scanner. Participants responded correctly in the control condition on $96.7 \%$ of the trials $(\mathrm{SD}=0.019)$, in the low overlap condition on $94.23 \%$ of the trials ( $\mathrm{SD}=0.049)$, and in the high overlap condition on $90.93 \%$ of the trials $(\mathrm{SD}=0.102)$, A repeated measures ANOVA revealed a significant main effect of condition $\left(F_{(2,16)}=4.00 ; P=0.028\right.$;

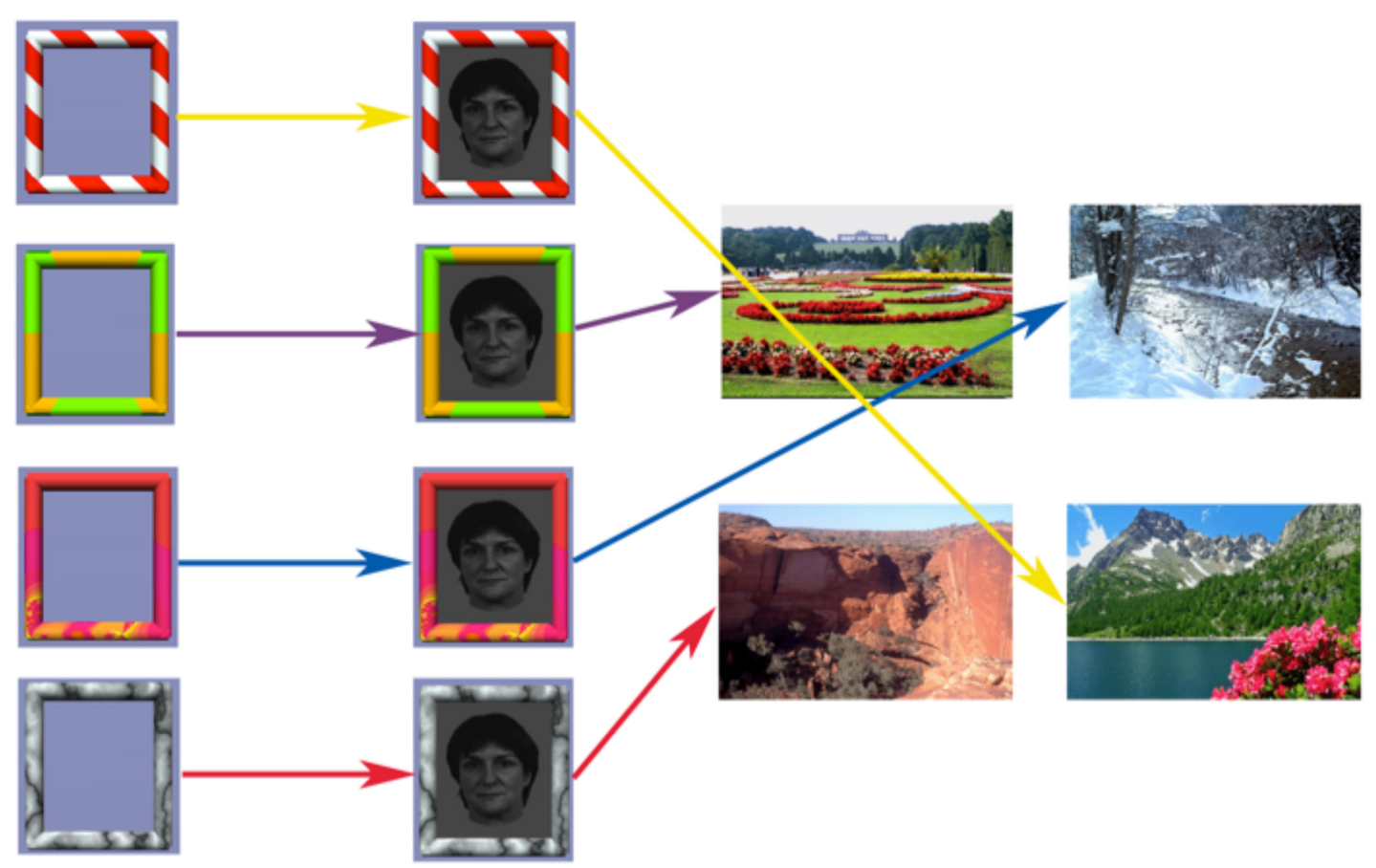

Figure 3. One of four sets of stimuli from the high overlap condition. Each frame and face was associated with four scenes, and the correct response was dependent on the frame initially shown. There were 16 overlapping sequences in the high overlap condition. This condition had the highest degree of overlap between sequence elements and the lowest level of predictability. 


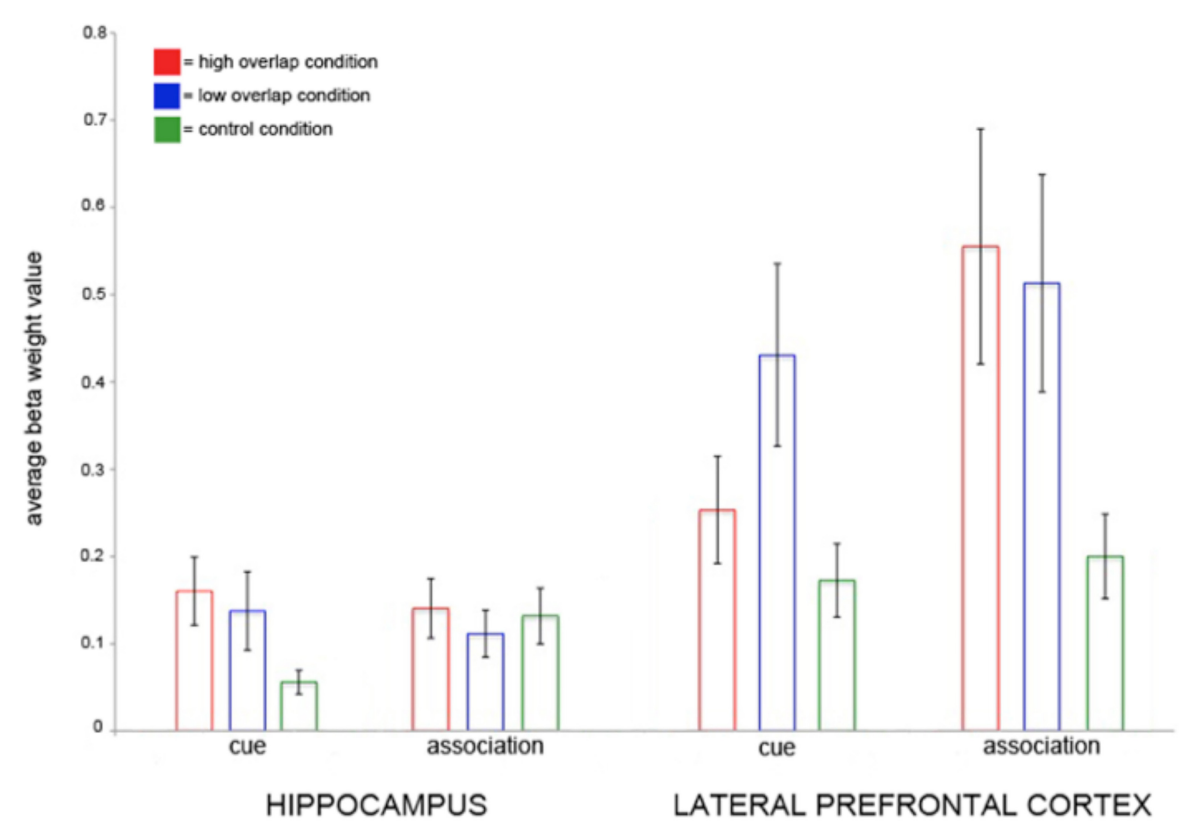

Figure 4. Beta weights from each condition at both time points of interest (Cue and Association). The conditions are presented in order of relational load within ROI and timepoint: high overlap (red), low overlap (blue), and control (green).

$\left.\eta_{p}^{2}=0.20\right)$. Post-hoc tests revealed that the main effect of condition was driven by differences in accuracy between the high overlap and control conditions $(P=0.035$, Fisher's Least Significant Difference (LSD) post-hoc test). No significant differences in percentage correct were found between the low and the high overlap conditions, indicating that the two overlapping sequences were performed with comparable accuracy $(P=0.156$, Fisher's LSD posthoc test).

Mean reaction time for the control condition was $1.45 \mathrm{sec}$ $(\mathrm{SD}=0.404)$, for the low overlap condition was $1.55 \mathrm{sec}(\mathrm{SD}=$ $0.5116)$, and for the high overlap condition was $1.56 \mathrm{sec}(\mathrm{SD}=$ 0.19). A repeated measures ANOVA revealed a significant main effect of condition on reaction time $\left(F_{(2,16)}=8.811, P=0.001\right.$; $\left.\eta_{p}^{2}=0.355\right)$. Post-hoc tests revealed that the main effect of condition was driven by differences between the overlapping and the control conditions. Responses in the control condition were significantly faster than in the low overlap condition $(P=0.01$; Fisher's LSD post-hoc test) and the high overlap condition $(P=0.002$; Fisher's LSD post-hoc test). The two overlapping conditions did not differ from one another in reaction time $(P=0.097$, Fisher's LSD post-hoc test).

\section{Functional MRI data}

We used fMRI to assess activation during sequence retrieval in regions of interest: the $\mathrm{HC}$, the dorsolateral PFC, and the ventrolateral PFC. To facilitate interpretation of our results, we averaged the beta values for each subject in each condition within ROIs and determined the overall mean beta value per condition at each timepoint and each region of interest (see Figs. 4 and 5 for bar

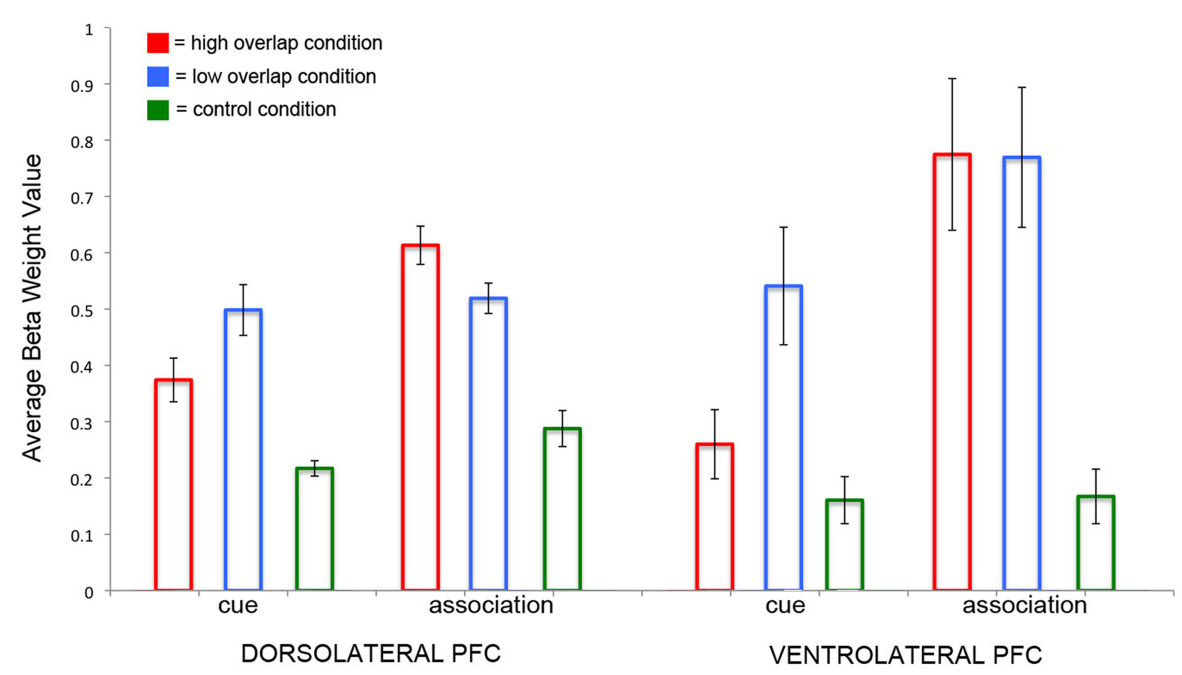

Figure 5. A graph showing the average beta weight values for the dorsolateral and ventrolateral PFC during the cue and association periods. Although the ROI used for the small volume correction analysis was comprised of the whole lateral PFC, we discussed the results from this analysis separately for dorsolateral and ventrolateral PFC and therefore show their individual beta weight values. 
Table 1. Small volume correction analysis complete results

\begin{tabular}{|c|c|c|c|c|}
\hline Condition & Region of interest & $\begin{array}{l}\text { Cluster size } \\
\text { (voxels) }\end{array}$ & $t$-value & $\begin{array}{l}\text { MNI peak } \\
\text { coordinates }\end{array}$ \\
\hline \multirow[t]{4}{*}{ High overlap > control association } & Right HC (body) & 98 & 5.27 & $24-28-8$ \\
\hline & Right dorsolateral PFC & 2710 & 9.41 & 482628 \\
\hline & Left dorsolateral PFC & 468 & 4.80 & -562824 \\
\hline & Left ventrolateral PFC & 261 & 8.92 & $-3424-6$ \\
\hline \multirow[t]{3}{*}{ Low overlap > control cue } & Left HC (tail) & 43 & 4.62 & $-12-360$ \\
\hline & Right HC (tail) & 20 & 3.62 & $18-340$ \\
\hline & Right dorsolateral PFC & 1510 & 7.35 & 481832 \\
\hline \multirow[t]{2}{*}{ Low overlap > control association } & Right dorsolateral PFC & 2829 & 7.05 & 501432 \\
\hline & Left dorsolateral PFC & 223 & 4.78 & -543426 \\
\hline Control > high overlap association & Left ventrolateral PFC & 48 & 3.93 & -521012 \\
\hline Control > low overlap association & Left ventrolateral PFC & 27 & 3.53 & -541012 \\
\hline
\end{tabular}

graphs of beta averages within the ROI masks used in the small volume correction analyses). In addition, we examined activation at the whole brain level in an exploratory analysis. For both analyses, six contrasts were analyzed: the high and low overlap conditions compared to control, the high and low overlap conditions compared to each other, and the control condition compared to the high and low overlap conditions. The results are presented separately for the High Overlap, Low Overlap, and Control conditions. Results are summarized in Tables 1 (ROI based) and 2 (Whole brain).

\section{Small volume correction analyses}

High overlap condition. In the high overlap condition, the frame indicated to the participant which set of stimuli the trial belonged to but it was not until the face was shown within the frame that participants were able to think about which scene would accurately complete the sequence. The results from our small volume correction analyses showed that the high overlap condition was associated with increased activation in the posterior body of the HC compared to the control condition during the association period (see Fig. 6).

Table 2. Whole brain analysis complete results

\begin{tabular}{|c|c|c|c|c|}
\hline Condition & Brain region & $\begin{array}{l}\text { Cluster size } \\
\text { (voxels) }\end{array}$ & $t$-value & $\begin{array}{l}\text { MNI peak } \\
\text { coordinates }\end{array}$ \\
\hline \multirow[t]{4}{*}{ High load > control cue } & Right intraparietal sulcus & 1472 & 3.99 & $26-7030$ \\
\hline & Left caudate & 888 & 5.45 & $-81-3$ \\
\hline & Left cuneus & 683 & 4.98 & $-18-5826$ \\
\hline & Left intraparietal sulcus & 440 & 5.23 & $-30-6634$ \\
\hline \multirow[t]{3}{*}{ High load > control association } & Right insula & 27,123 & 10.20 & 28222 \\
\hline & Bilateral anterior cingulate cortex & 1636 & 8.76 & 43042 \\
\hline & Right cerebellum & 1155 & 6.95 & $4060-32$ \\
\hline \multirow[t]{2}{*}{ High load > low load association } & Bilateral posterior cingulate cortex & 2547 & 5.49 & $0-2030$ \\
\hline & Left angular gyrus & 469 & 6.36 & $44-7034$ \\
\hline \multirow[t]{5}{*}{ Low load > control cue } & Left inferior parietal lobule & 8752 & 7.79 & $-34-5040$ \\
\hline & Left lateral occipital gyrus & 3432 & 6.72 & $-34-86-2$ \\
\hline & Right cerebellum & 3102 & 7.98 & $20-36-48$ \\
\hline & Right dorsolateral PFC & 1514 & 6.67 & 382024 \\
\hline & Right supplementary motor area & 1119 & 6.97 & 21454 \\
\hline Low load $>$ high load cue & Left middle occipital gyrus & 1726 & 7.79 & -30962 \\
\hline \multirow[t]{8}{*}{ Low load > control association } & Right angular gyrus & 5406 & 8.55 & $32-5438$ \\
\hline & Right fusiform gyrus & 4075 & 7.65 & $36-42-26$ \\
\hline & Right dorsolateral PFC & 2881 & 7.61 & 521828 \\
\hline & Right inferior parietal lobule & 584 & 7.22 & $30-5232$ \\
\hline & Left dorsolateral PFC & 1691 & 6.62 & -402220 \\
\hline & Bilateral supplementary motor area & 589 & 5.64 & 01448 \\
\hline & Left angular gyrus & 586 & 7.09 & -365240 \\
\hline & Right cerebellum & 615 & 6.74 & $2036-48$ \\
\hline \multirow[t]{4}{*}{ Control > low load cue } & Left middle temporal cortex & 808 & 4.65 & $-58-5418$ \\
\hline & Right middle temporal cortex & 761 & 5.98 & $62-18-10$ \\
\hline & Right angular gyrus & 594 & 5.19 & $52-6428$ \\
\hline & Right dorsomedial PFC & 538 & 5.01 & 66212 \\
\hline \multirow[t]{5}{*}{ Control > high load association } & Bilateral dorsomedial PFC & 3648 & 8.32 & 0584 \\
\hline & Left insula & 3028 & 5.91 & $-40-14-4$ \\
\hline & Right supramarginal gyrus & 2917 & 6.31 & $60-3642$ \\
\hline & Bilateral middle cingulate gyrus & 1945 & 7.38 & $0-2444$ \\
\hline & Left middle temporal cortex & 482 & 5.09 & $-62-404$ \\
\hline \multirow{4}{*}{ Control > low load association } & Left insula & 2656 & 8.59 & $-40-12-4$ \\
\hline & Right insula & 2544 & 8.75 & $40-16-4$ \\
\hline & Bilateral dorsomedial PFC & 2491 & 6.90 & 0586 \\
\hline & Left middle cingulate gyrus & 1561 & 6.38 & $-4-2246$ \\
\hline
\end{tabular}


HIGH OVERLAP REGION OF INTEREST RESULTS
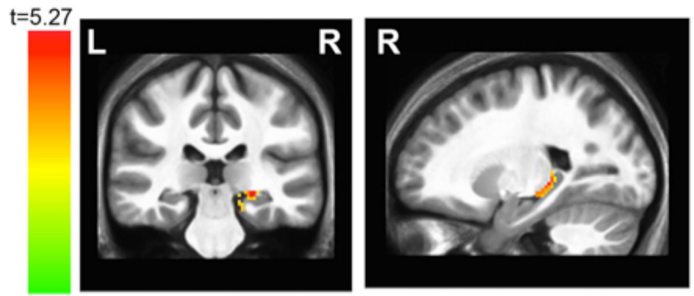

High Overlap > Control : Association Period
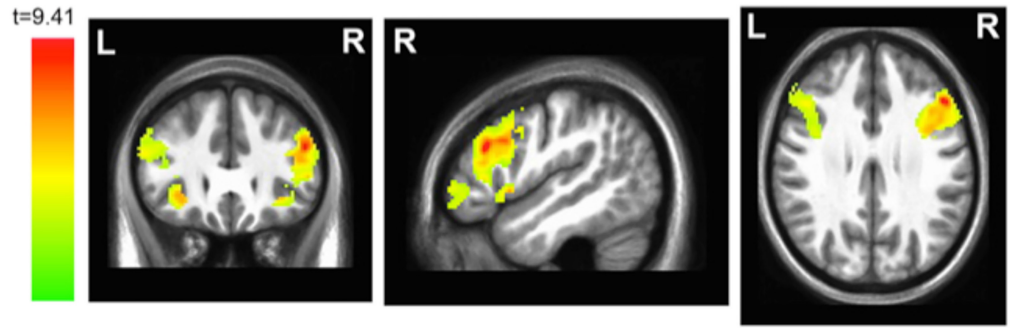

High Overlap > Control : Association Period

Figure 6. (Top row) The posterior $\mathrm{HC}$ showed increased activation at time points with increased predictability. In the high overlap condition, at the association time point, the participant was able to accurately predict how to complete the sequence. (Bottom row) In the lateral PFC, the high overlap condition showed increased activation at the association time point. This may be due to the increased level of cognitive control required to inhibit other associations irrevelant to the current trial. scenes. When the contrasts created for the low overlap cue period were compared to those of the control cue period, significantly more activation was seen in the tail of the left and right HC in the low overlap condition (Fig. 7). We expected to see activation in the HC during this condition because the cue prompts retrieval of the two faces and scenes paired with it.

During the cue period, the low overlap condition also showed increased activation in the right dorsolateral PFC compared to the control condition. During the association period, the low overlap condition showed increased activation in the right and left dIPFC compared to control. During both of these time points, the dIPFC may be active due to the fact that information is being obtained revealing the appropriate neural representations needed to make a correct response.

Control condition. The control showed increased activation in a small posterior region of the left ventrolateral PFC
During this timepoint, the high overlap condition also showed increased activation in the right dorsolateral PFC, the left dorsolateral PFC, and the left ventrolateral PFC compared to the control condition. At this point, there may be interference from overlapping elements, prompting recruitment of the PFC (Irlbacher et al. 2014 for review; Jonides and Nee 2006). In addition, at this stage, the participant has enough information to determine which scene accurately completes the sequence. The dIPFC may be needed at this timepoint to activate the regions needed to correctly respond.

There were no significant differences between the low and high overlap conditions during the cue period in either the MTL or lateral PFC. In addition, the high overlap and control conditions did not show differences in activation in either region of interest during the cue period. This may be due to the fact that in the high overlap condition, the cue period enables the participant to know which condition the trial is but does not allow the participant to exclude any of the four upcoming scenes.

Low overlap condition. Presentation of the picture frame in the low overlap condition enables the participant to narrow down the upcoming sequence from 33 possible sequences to two sequences. At this point in the sequence, the participant can predict that one of two possible faces will follow and two of four possible

\section{LOW OVERLAP REGION OF INTEREST RESULTS}
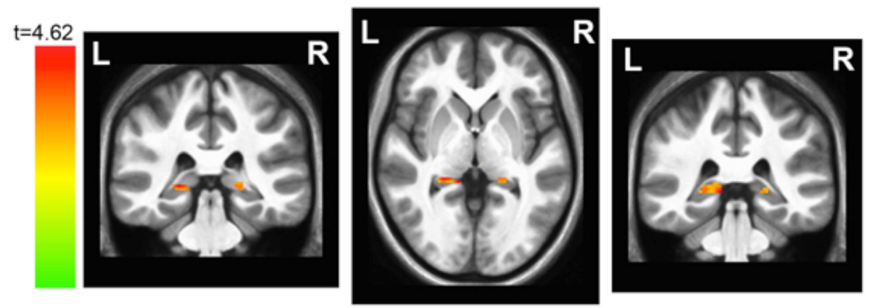

Low Overlap > Control : Cue Period
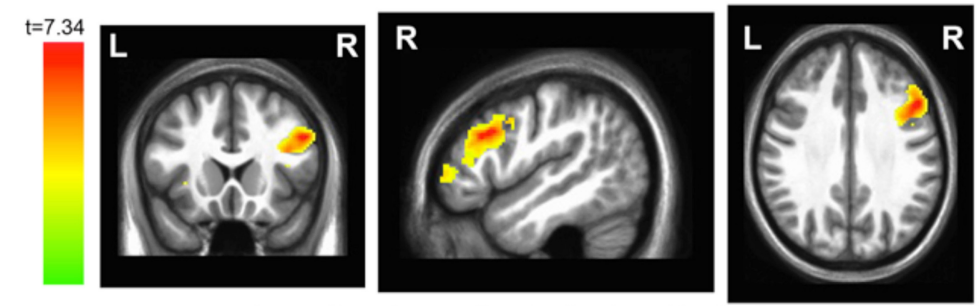

Low Overlap > Control : Cue Period
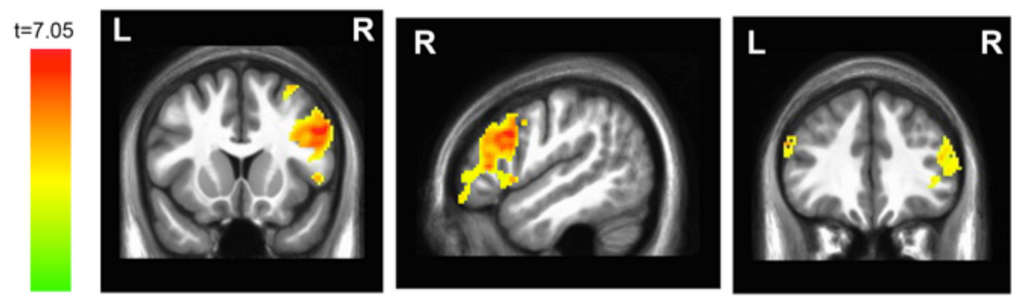

Low Overlap > Control : Association Period

Figure 7. (Top row) During the cue period, the participant was able to decrease the number of potential upcoming sequences in the low overlap condition to two. This time point also showed increased activation in the posterior HC. (Middle and bottom rows) The low overlap condition showed increased activation in the right dorsolateral PFC that coincided with the participant's increased ability to predict the upcoming sequence. 

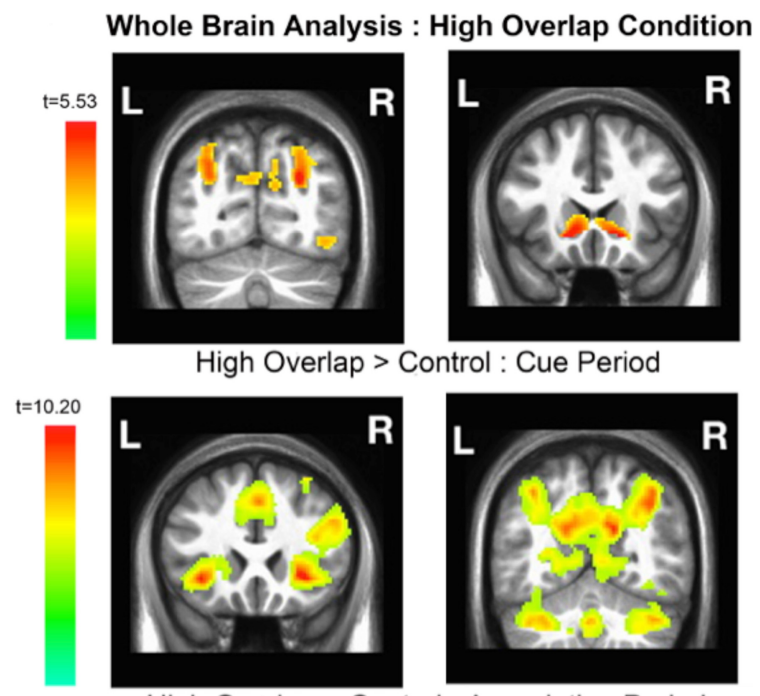

High Overlap > Control : Association Period
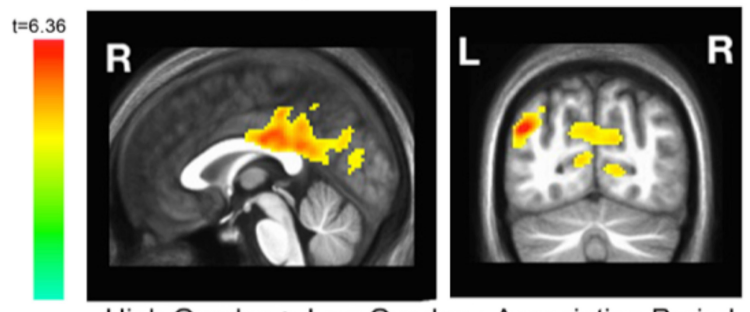

High Overlap > Low Overlap : Association Period

Figure 8. (Top row) Whole brain analysis revealed additional regions of activation in the high overlap condition compared to the control during the cue period. (Middle and bottom rows) During the association period, whole brain analyses revealed increased activation in the anterior cingulate cortex, the insula, and dorsolateral PFC, all regions that are part of the cognitive control network, in the high overlap condition compared to the control condition. In addition, compared to the low overlap condition, the high overlap condition showed increased activation in parietal regions including the precuneus, the cuneus, and the angular gyrus. compared to both the low overlap and high overlap conditions during the association period. There were no other differences between the control and overlapping conditions in any region of interest.

\section{Exploratory whole brain analysis}

To examine additional brain regions involved in disambiguating between overlapping elements in our task, we compared the contextual cue and association segments of the trials between the three conditions in an exploratory whole brain analysis. Table 2 shows a list of the complete results from the whole brain exploratory analysis. All reported results have been defined by cluster mass with a threshold set to $P<0.01$.

High overlap condition. During the cue period, the high overlap condition showed increased activation in bilateral intraparietal sulcus, left caudate, and left cuneus compared to the control condition. During the association period, regions that are part of the cognitive control network showed increased activation in the high overlap condition compared to the control condition including the insula, the dlPFC, and the anterior cingulate cortices (Cole and Schneider 2007). Compared to the low overlap condition at the same time point, the high overlap condition showed increased activity in the posterior cingulate and angular gyrus (see Fig. 8).

Low overlap condition. During the cue period, the low overlap condition had similar regions of activation that the high overlap condition had during the association period when both were compared to control. The low overlap condition showed increased activation in regions that are part of the cognitive control network including the dIPFC and the SMA. During the association period, the low overlap condition showed increased activation in bilateral angular gyrus, fusiform gyrus, inferior parietal lobule, and SMA compared to the control condition (see Fig. 9).

Control condition. The control condition showed increased activation in regions that are part of the default mode network during both

Whole Brain Analysis : Low Overlap Condition
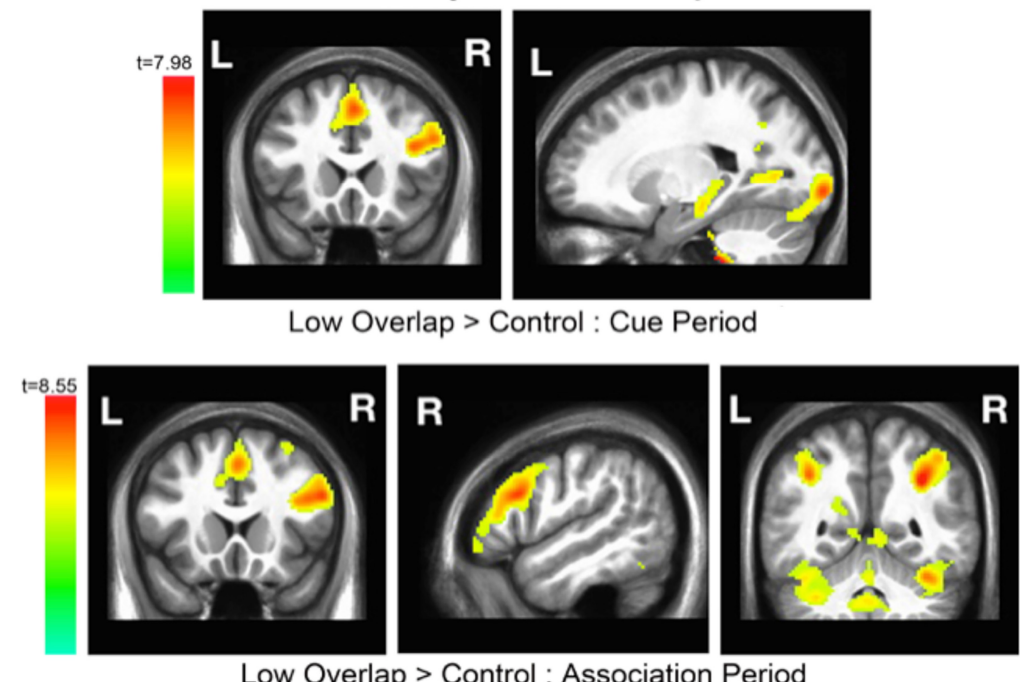

Low Overlap > Control : Association Period

Figure 9. (Top row) In the cue period of the low overlap condition, regions associated with memory retrieval (HC and angular gyrus) and cognitive control (dIPFC and SMA) showed increased activation compared to the control condition. (Bottom row) In the association period, the patterns of increased activation in the high and low overlap conditions compared to control were more similar than at the cue period. At this time point the low overlap condition showed increased activation in the SMA, the right dIPFC, and the inferior parietal lobules. 
the cue and association periods compared to the overlapping conditions. These regions included the medial PFC and the temporal cortex. In addition the control condition showed increased activity in the insula and middle cingulate gyrus compared to the overlapping conditions during the association period.

\section{Discussion}

We manipulated the number of overlapping elements in a contextdependent sequencing task to examine the effects of degree of overlap and sequence predictability during memory retrieval. Our results showed that during retrieval of sequences with overlapping elements, the $\mathrm{HC}$ was more active at time points that enabled prediction of a correct response compared to sequences without overlapping elements at the same time points. In addition, the dorsolateral PFC was active when the pool of possible upcoming stimuli was reduced and the proper set of sequences could be delineated. The ventrolateral PFC, on the other hand, was active when responses had to be inhibited due to interference from overlapping associations.

\section{Overlapping sequences and sequence predictability modulated hippocampal activity in a context-dependent memory retrieval task}

During the contextual cue period, the small volume correction analyses showed increased activation in the left and right hippocampal tail when the low overlap condition was compared to the control condition. In the low overlap condition, there were four sets of sequences with stimuli overlapping within each set. The picture frame signaled to which set of sequences the trial belonged. Because the task was well learned, the frame (cue) enabled retrieval of the two faces and scenes associated with that frame through activation in the posterior HC. Although the contextual cue in the control also enabled retrieval of its associated sequence, the cue in the low overlap condition had more stimuli associated with it than the control condition, which may have resulted in the increased activity in posterior $\mathrm{HC}$ seen at this time point. This result was similar to that seen in Brown et al. (2010) in which the first hallway of an overlapping maze served as the contextual cue during which the participant was able to think ahead to the overlapping hallway and plan which way to turn. During the cue period of the overlapping mazes, Brown et al (2010) found increased activation in the posterior HC compared to the nonoverlapping mazes.

During the association phase of the trial, when a face was shown within the picture frame, the cue was paired with the stimulus that determined the correct response. At this point in all conditions, the participant should have been able to predict the scene corresponding to that face-frame pairing. In the high overlap condition, this meant a narrowing down from four possible options to one. When the high overlap condition was compared to the control condition during the association phase, increased activation was seen in the body of the right HC. The increased activity in the body of the HC may have resulted from item and context information being combined at this phase, a process that involves the HC (Komorowski et al. 2009; Eichenbaum et al. 2012). In a human neuroimaging study, using representational similarity analysis to examine fMRI data, Collin et al. (2015) determined that the mid-portion of the $\mathrm{HC}$ was active during presentation of directly integrated event-pair associations that were part of a multiple event narrative. Unlike the posterior $\mathrm{HC}$, which was active only during directly associated events, the body of the $\mathrm{HC}$ became active when multiple events were linked together to form a narrative. Interestingly, when participants in our study were asked to describe their learning strategies regarding the high overlap condition many stated they created stories that bound the frame, face, and scene together to keep track of the multiple overlapping sequences.

\section{Different regions of lateral PFC responded when overlapping elements in a context-dependent memory task increased}

Considerable converging evidence indicates that the role of the PFC in memory retrieval is strategic control of processes in other brain areas and the compiling of features of related memories to provide a context for related experiences (Buckner and Wheeler 2001; Miller and Cohen 2001; Dobbins et al. 2002; Blumenfeld and Ranganath 2007; Kuhl and Wagner 2009; Preston and Eichenbaum 2013). We found differences in activation of the dorsolateral PFC that were dependent on condition and phase of the trial. The overlapping conditions had a larger number of associations related to each stimulus (high $>$ low $>$ control) and previous research suggests the anterior dlPFC is involved in creating and integrating complex relationships between stimuli (Kroger et al. 2002; Blumenfeld and Ranganath 2006; Murray and Ranganath 2007). In addition, another proposed role of the dlPFC is top-down activation of the neural representations appropriate for completing a task (Rowe et al. 2000). During the cue period, the low overlap condition showed increased right dlPFC activation compared to the control condition. The cue period in the low overlap condition prompted recall of two possible sequences whereas the control condition required retrieval of a single sequence. In the high and low overlap conditions, we found increased bilateral dlPFC activation in the association phase of the task compared to the control condition. The association phase in both the high and low overlap conditions enabled recall of the correct outdoor scene to complete the sequence. Therefore, it may be the recall of multiple associated stimuli combined with increased relational complexity inherent to a task with overlapping associations that required the dorsolateral PFC.

During the association phase of the task, the high overlap condition showed increased activation in the left anterior and middle ventrolateral PFC. Previous research has shown recruitment of the anterior vlPFC during tasks requiring control over memory access (Barredo et al. 2016) and recruitment of left vlPFC in particular when there is interference from overlapping associations (Nee et al. 2007; Irlbacher et al. 2014). At the association time point in the high overlap condition, the participant was required to recall the correct scene that completed the sequence and inhibit the three scenes irrelevant to the trial. This result supports the proposed role of vlPFC in activating correct sequences or associations while also inhibiting irrelevant associations (Petrides 2002; Badre and Wagner 2005, Nee et al. 2007). Barredo et al. (2015) showed that anterior vlPFC is part of a functionally connected network that includes the $\mathrm{HC}$ among other regions.

\section{Whole brain analyses revealed regions in the cognitive control network and the default mode network active for different conditions during this task}

In contrasts comparing the low overlap and control conditions, regions that are considered part of the cognitive control network, including the dorsolateral PFC and the rostral SMA, showed increased activation during both the cue and association periods. When the high overlap condition was compared to the control at the association time point, increased activation was seen in the dorsolateral PFC, the anterior cingulate cortex, and the anterior insula, also regions considered part of the cognitive control network. 
The most striking result from comparisons between the control and overlapping conditions was the strong activation in the dorsomedial prefrontal cortices (dmPFC) in the control condition. At the cue, the activation in dmPFC was increased compared to control but by the association period there was a large region of activation in the dmPFC compared to both the high and low overlap conditions. The medial PFC is a major hub of the default mode network (Buckner et al. 2008).

\section{Limitations and conclusions}

In this study we sought to better understand the neural response to disambiguation of sequences with varying degrees of overlap and the interplay of predictability in episodic retrieval and its accompanying neural response. Initially the task was constructed like a more typical sequence in which the picture frame was shown, then a photograph of a face (without the frame), followed by the choice of four scenes. We sought to replicate animal studies in which context is temporally separated from its associations and determines the appropriate response (Komorowski et al. 2009; Navawongse and Eichenbaum 2013). However, when we piloted this task, participants were not able to learn the high overlap condition when the frame was separated from the face. We sought to even out the task difficulty and accuracy scores between conditions by enclosing the face with the frame in the second time point of the trial. Participants were able to learn the high overlap condition with this change but it made our results more difficult to interpret because the frame, or cue, was no longer separate from its associated face.

In interpreting the data, knowing the strategies participants used to remember the overlapping associations may have been enlightening. We did not conduct a formal interview of participants following the testing session on day two, but in the future would add this to similar studies. In addition, it would have been interesting to ask participants to return $1 \mathrm{wk}$ after scanning to test how much they retained from the task, to see if there were differences in retention between the high and low overlap conditions and if neural activation was correlated with sequence retention.

When we designed this task we expected to see linear increases in activation that were dependent on the degree of overlap in each condition. We thought that because the high overlap condition had four times as many overlapping associations between each sequence than the low overlap condition we would see the highest level of activation in the MTL and PFC in the high overlap condition. However, what we found was that the extent to which stimuli revealed information about the upcoming sequence and increased the predictability of a correct response was the critical factor that determined which brain regions were active. Both our small volume correction and whole brain analyses demonstrated patterns of activation dependent on previously learned sequence predictability at each time point. In the low overlap condition, activation in the HC occurred during the cue period at which time the participant was able to whittle down the potential upcoming sequence from a large pool of possibilities to two sequences. In the high overlap condition, it was not until the association period that the HC became active and it was not until this time point that the participant was provided enough information to predict the upcoming sequence. At the association time point, participants were able to predict the correct response in both the high and low overlap conditions and both conditions showed similar patterns of activation in the dorsolateral PFC. The dIPFC may work with the HC to decipher the correct way to complete a sequence in a task with multiple possibilities by indicating the correct set of sequences and conveying that information back to the medial temporal lobe. The vlPFC may then inhibit the incorrect responses, a process integral to the participant ultimately accurately completing the sequence.

\section{Materials and Methods}

\section{Participants}

Participants were 18-34 yr old adults recruited from the Boston University community. Written informed consent was obtained from each participant before enrollment in accordance with the experimental protocol approved by both the Partners Human Research Committee and the Boston University Charles River Campus Institutional Review Board.

Twenty-one subjects underwent training followed $1 \mathrm{~d}$ later by testing in the fMRI scanner. Two subjects were omitted from the data analysis due to excessive motion in the scanner. Two subjects were excluded due to accuracy scores more than two standard deviations below the mean. Seventeen subjects ( 11 females) were included in the final data analysis (mean age $\pm S D=22.84 \pm 4.92$ ).

\section{Procedure}

\section{Task summary}

After observing a frame and a face, the participant's task was to select the scene that correctly completed the sequence. The initial time point was the picture frame, or contextual cue, which indicated to the participant which condition the trial was. In the low overlap condition this enabled the participant to narrow down the possible upcoming sequences from 16 down to two. In the high overlap condition this indicated to the participant that there were four possible upcoming sequences, out of a possible 16 in that condition. In the control condition, because there was only one sequence, the participant knew at this time point what the correct response was. The next time point was the presentation of the face in the picture frame, or the association period. At this point in all three conditions, the participant should be able to predict the correct response. In the low overlap condition this meant eliminating one of the two possibilities. In the high overlap condition this meant narrowing down the possibilities from four to one. below.

A more detailed description of the task design is provided

\section{Task description}

The task was programmed using E-Prime 2.0 software (Psychology Software Tools 2012). A picture frame appeared on the computer monitor for $2 \mathrm{sec}$ followed by a $4 \mathrm{sec}$ blank delay. Then a black and white photograph within the same picture frame was shown on the screen for $2 \mathrm{sec}$ followed by a 4 sec blank delay. Then four photographs of outdoor scenes were presented on the screen for $4 \mathrm{sec}$ and the participant was instructed to choose one of the scenes using the number pad on the keyboard. The correct response was determined by which picture frame and face were shown in the beginning of the sequence. During training the participants received feedback based on their response-either "Correct" or "Incorrect" appeared on the screen.

Stimuli consisted of images of 13 picture frames, each distinguishable by its unique color, texture, and/or pattern, 13 black and white photographs of faces with neutral expressions placed within the picture frame, and 16 color photographs of outdoor scenes. Each trial consisted of the contextual cue period (picture frame), the association period (photograph of face within the frame), and the test phase (photographs of four outdoor scenes) (see Fig. 1 ). The spatial location of the four outdoor scenes shown on the screen changed between trials and was counterbalanced across trials.

The task consisted of three conditions that varied by degree of overlap between sequences. The high overlap condition consisted of one set of sequences sharing four frames, four faces, and four scenes for a total of 16 frame/face/scene combinations. In this condition the stimuli included four unique frames, four unique faces, and four unique scenes.

The low overlap condition consisted of four sets of sequences sharing two frames, two faces, and two scenes. Each set consisted of four sequences for a total of 16 frame/face/scene combinations. 
In this condition the stimuli included eight unique frames, eight unique faces, and eight unique scenes.

The control condition consisted of a single unique frame/ face/scene sequence.

Training one day prior to scanning. During the training phase of the experiment, research participants learned each contextual cue (frame) and its corresponding association-test sequence (face-scene). During the first two trials of training, only the correct outdoor scene for that frame and face was shown during the test phase. In all subsequent trials, four outdoor scenes were presented and left on the screen during the test phase until the participant made a response. The participant used a number pad to indicate which of the four scenes was correct for that trial and was then given feedback ("Correct" or "Incorrect") that appeared on the computer screen for $1.5 \mathrm{sec}$. If the response was incorrect, the participant was not told what the correct answer was.

During training, participants learned one new sequence per training run. In all training runs after the first run, sequences previously learned were repeated for additional rehearsal. Training runs were balanced so that each unique sequence was presented a total of eleven times during training.

Testing during fMRI scanning. Participants returned for fMRI scanning $1 \mathrm{~d}$ after training. While in the scanner, participants completed six runs of 24 trials each. Each run included eight trials per condition that were identical to the training trials except that there was a $4 \mathrm{sec}$ time limit for responding and participants were not given feedback on their performance. The three conditions were interspersed within each run. The order of trials was counterbalanced across runs, and the order of runs was randomized across participants. There were 48 trials of each condition per participant.

Image acquisition. Images were acquired using a 3 Tesla Siemens MAGNETOM TrioTim scanner with a 32 channel Tim Matrix head coil located at the Athinoula A. Martinos Center for Biomedical Imaging, Massachusetts General Hospital, Harvard Medical School. A high resolution T1-weighted multiplanar rapidly acquired gradient echo (MP-RAGE) structural scan was acquired using Generalized Autocalibrating Partially Parallel Acquisitions (GRAP$\mathrm{PA})\left(\mathrm{TR}=2530 \mathrm{msec} ; \mathrm{TE}=3.4 \mathrm{msec}\right.$; flip angle $=7^{\circ}$; slices $=176$; resolution $=1 \mathrm{~mm}$ isotropic). T2*-weighted BOLD images were acquired using an Echo Planar Imaging sequence $(\mathrm{TR}=2000 \mathrm{msec}$; $\mathrm{TE}=30 \mathrm{msec} ;$ flip angle $=85^{\circ}$; slices $=33$; resolution $=3.4 \times 3.4 \times$ $3.4 \mathrm{~mm}$, interslice gap of $0.5 \mathrm{~mm}$ ). Functional image slices were aligned parallel to the long axis of the HC.

Image preprocessing. Functional imaging data were preprocessed using the SPM8 software package (Statistical Parametric Mapping, Wellcome Department of Cognitive Neurology, London). All BOLD images were first reoriented so the origin (i.e., coordinate $x$ y $z=[0,0,0])$ was at the anterior commissure. The images were then corrected for differences in slice timing and were realigned to the first image collected within a series. Motion correction was conducted and included realigning and unwarping the BOLD images (Andersson et al. 2001). The high-resolution structural image was then coregistered to the mean BOLD image created during motion correction and segmented into white and gray matter images. The bias-corrected structural image and coregistered BOLD images were spatially normalized into standard MNI space using the Diffeomorphic Anatomical Registration Through Exponentiated Lie (DARTEL) algebra algorithm (Ashburner 2007) for improved intersubject registration. BOLD images were resampled during normalization to $2 \mathrm{~mm}^{3}$ isotropic voxels and smoothed using a 6 $\mathrm{mm}$ full-width at half-maximum Gaussian kernel. The normalized structural images of all 17 participants were averaged after normalization for displaying overlays of functional data.

Behavioral analyses. A repeated measures ANOVA was run to compare accuracy and reaction time between the low overlap, high overlap, and control conditions. When significant differences were found between the three groups, a Fisher's LSDs post-hoc test was run to determine which groups significantly differed from one another. Analyses were completed using PASW Statistics (PASW Statistics for Windows, Version 18.0. Chicago: SPSS Inc.).

fMRI analyses. Nine regressors of interest were created for each participant to model the fMRI data. Separate regressors were created for the following three elements in each condition: contextual cue (presentation of the frame), association phase, (presentation of the face in the frame), and test phase (presentation of the four outdoor scenes). Incorrect trials and the six motion parameters calculated during motion correction were added to the model as additional covariates of noninterest. Regressors from the task were constructed as a series of square waves or "boxcars." Boxcar onsets were defined by the onset of each event and extended for the duration of the event $(2 \mathrm{sec}$ for the contextual cue and association phases, $4 \mathrm{sec}$ for the test phase). These parameters were convolved with the canonical hemodynamic response function in SPM8.

In total, six primary contrasts were created: the low and high overlap conditions each compared to the control conditions during the cue and association periods and the low and high overlap conditions compared to each other during the cue and association periods. The model was then analyzed using the general linear model approach.

All six primary contrasts were tested at the group level.

Small volume correction analyses. Due to our a priori hypothesis that hippocampal and prefrontal cortical activity would be modulated by degree of overlap, we defined regions of interest (ROIs) in the MTL and the PFC to perform voxelwise analyses within these areas. The MTL ROI consisted of the HC and parahippocampal gyrus in both hemispheres, as defined by the aal library within the Wake Forest University (WFU) pick atlas, a standard set of anatomical definitions defined by hand on a single brain which matched the MNI/ICBM templates (Tzourio-Mazoyer et al. 2002). The PFC ROI consisted of the dorsolateral PFC, defined as the middle frontal gyrus and Brodmann areas 9 and 46, and the ventrolateral PFC, defined as the inferior frontal gyrus and Brodmann areas 44, 45, and 47. The aal and Brodmann area libraries within the WFU pick atlas were used to define the regions. All ROIs were defined bilaterally.

All region of interest, group-level analyses assessed statistical significance on the basis of cluster mass, with the cluster-defining threshold set to the nominal $P<0.01$ level. Corrected $P$ values were determined using permutation testing (FSL's randomise; 5000 iterations), and results were thresholded at corrected $P<0.01$. For onesample $t$-tests, each iteration randomly sign-flipped individual subjects' contrast coefficient maps and added the resulting maximum cluster mass to the empirical null-hypothesis distribution.

Whole-brain analyses. All whole-brain, group-level analyses assessed statistical significance on the basis of cluster mass, with the clusterdefining threshold set to the $P<0.01$ level. Corrected $P$ values were determined using permutation testing (FSL's randomise; 5000 iterations), and results were thresholded at corrected $P<0.01$. For one-sample $t$-tests, each iteration randomly sign-flipped individual subjects' coefficient maps. Clusters were defined as having faces or edges or corners that touched and a minimum of 20 voxels. Peak activations within each cluster of activation were identified using AFNI software package (http://afni.nimh.nih.gov/afni). If a specific region of activity had multiple peaks within a cluster, the peak with the highest $t$-value was reported. Brain regions were identified using MRIcron software and brain atlases (Damasio 2005; Petrides 2005) .

\section{Acknowledgments}

This work was supported by the National Institutes of Health grant P50 MH0941263 and the Office of Naval Research Multidisciplinary University Research Initiative (MURI) grant N00014-16-1-283. We thank Dr. Joseph McGuire for helpful discussions regarding analysis. 


\section{REFERENCES}

Agster KL, Fortin NJ, Eichenbaum H. 2002. The hippocampus and disambiguation of overlapping sequences. J Neurosci 22: 5760-5768.

Andersson JL, Hutton C, Ashburner J, Turner R, Friston K. 2001. Modeling geometric deformations in an EPI time series. Neuroimage 13: 903-919.

Ashburner J. 2007. A fast diffeomorphic image registration algorithm. Neuroimage 38: 95-113.

Badre D, Wagner AD. 2005. Frontal lobe mechanisms that resolve proactive interference. Cereb Cortex 15: 2003-2012.

Badre D, Wagner AD. 2007. Left ventrolateral prefrontal cortex and the cognitive control of memory. Neuropsychologia 45: 2883-2901.

Bakker A, Kirwan CB, Miller M, Stark CE. 2008. Pattern separation in the human hippocampal CA3 and dentate gyrus. Science 319: 1640-1642.

Barredo J, Öztekin I, Badre D. 2015. Ventral fronto-temporo pathway supporting cognitive control of episodic memory retrieval. Cereb Cortex 25: $1004-1019$.

Barredo J, Verstynen TD, Badre D. 2016. Organization of cortico-cortical pathways support memory retrieval across subregions of the left ventrolateral prefrontal cortex. J Neurophysiol 116: 920-937.

Bennett IJ, Stark CE. 2016. Mnemonic discrimination relates to perforant path integrity: an ultra-high resolution diffusion tensor imaging study. Neurobiol Learn Mem 129: 107-112.

Blumenfeld RS, Ranganath C. 2006. Dorsolateral prefrontal cortex promotes long-term memory formation through its role in working memory organization. J Neurosci 26: 916-925.

Blumenfeld RS, Ranganath C. 2007. Prefrontal cortex and long-term memory encoding: an integrative review of findings from neuropsychology and neuroimaging. Neuroscientist 13: 280-291.

Brown TI, Ross RS, Keller JB, Hasselmo ME, Stern CE. 2010. Which way was I going? Contextual retrieval supports the disambiguation of well learned overlapping navigational routes. J Neurosci 30: 9414-7422.

Brown TI, Ross RS, Tobyne SM, Stern CE. 2012. Cooperative interactions between hippocampal and striatal systems support flexible navigation. Neuroimage 60: 1316-1330.

Brown TI, Whiteman AS, Aselcioglu I, Stern CE. 2014. Structural differences in hippocampal and prefrontal gray matter volume support flexible context-dependent navigation ability. J Neurosci 34: 2314-2320.

Buckner RL, Wheeler ME. 2001. The cognitive neuroscience of remembering. Nat Rev Neurosci 2: 624-634.

Buckner RL, Andrews-Hanna JR, Schacter DL. 2008. The brain's default network. Ann N Y Acad Sci 1124: 1-38.

Caplan JB, McIntosh AR, DeRosa E. 2007. Two distinct functional networks for successful resolution of proactive interference. Cereb Cortex 17: 1650-1663.

Chapados C, Petrides M. 2015. Ventrolateral and dorsomedial frontal cortex lesions impair mnemonic context retrieval. Proc Biol Sci 282: 20142555.

Cole MW, Schneider W. 2007. The cognitive control network: integrated cortical regions with dissociable functions. Neuroimage 37: 343-360.

Collin SH, Milivojevic B, Doeller CF. 2015. Memory hierarchies map onto the hippocampal axis. Nat Neurosci 18: 1562-1564.

Damasio H. 2005. Human brain anatomy in computerized images, 2 nd ed. Oxford University Press, Oxford, England.

D'Esposito M, Postle BR, Jonides J, Smith EE. 1999. The neural substrate and temporal dynamics of interference effects in working memory as revealed by event-related functional MRI. Proc Natl Acad Sci 96: 7514-7519.

Dobbins IG, Foley H, Schacter DL, Wagner AD. 2002. Executive control during episodic retrieval: multiple prefrontal processes subserve source memory. Neuron 35: 989-996.

Eichenbaum H. 2004. Hippocampus, cognitive processes, and neural representations that underlie declarative memory. Neuron 44: 109-120.

Eichenbaum H, Sauvage M, Fortin N, Komorowski R, Lipton P. 2012. Towards a functional organization of episodic memory in the medial temporal lobe. Neurosci Biobehav Rev 36: 1597-1608.

Favila SE, Chanales AJ, Kuhl BA. 2016. Experience-dependent hippocampal pattern differentiation prevents interference during subsequent learning. Nat Commun 7: 11066.

Frank LM, Brown EN, Wilson M. 2000. Trajectory coding in the hippocampus and entorhinal cortex. Neuron 27: 169-178.

Hasselmo ME. 2005. What is the function of hippocampal theta rhythm? Linking behavioral data to phasic properties of field potential and unit recording data. Hippocampus 15: 936-949.

Hsieh LT, Ranganath C. 2015. Cortical and subcortical contributions to sequence retrieval: Schematic coding of temporal context in the neocortical recollection network. NeuroImage 21: 78-90.

Irlbacher K, Kraft A, Kehrer S, Brandt SA. 2014. Mechanisms and neuronal networks involved in reactive and proactive cognitive control of interference in working memory. Neurosci Biobehav Rev 46: 58-70.

Jonides J, Nee DE. 2006. Brain mechanisms of proactive interference in working memory. Neuroscience 139: 181-193.
Kahn I, Davachi L, Wagner AD. 2004. Functional-neuroanatomic correlates of recollection: implications for models of recognition memory. J Neurosci 24: 4172-4180.

Komorowski RW, Manns JR, Eichenbaum H. 2009. Robust conjunctive item-place coding by hippocampal neurons parallels learning what happens where. J Neurosci 29: 9918-9929.

Komorowski RW, Garcia CG, Wilson A, Hattori S, Howard MW, Eichenbaum H. 2013. Ventral hippocampal neurons are shaped by experience to represent behaviorally relevant contexts. J Neurosci 33: 8079-8087.

Kroger JK, Sabb FW, Fales CL, Bookheimer SY, Cohen MS, Holyoak KJ. 2002. Recruitment of anterior dorsolateral prefrontal cortex in human reasoning: a parametric study of relational complexity. Cereb Cortex 12: $477-485$.

Kuhl BA, Wagner AD. 2009. Forgetting and retrieval. In Handbook of neuroscience for the behavioral sciences. John Wiley \& Sons.

Lacy JW, Yassa MA, Stark SM, Muftuler LT, Stark CE. 2011. Distinct pattern separation related transfer functions in human CA3/dentate and CA1 revealed using high-resolution fMRI and variable mnemonic similarity. Learn Mem 18: 15-18.

LaRocque KF, Smith ME, Carr VA, Witthoft N, Grill-Spector K, Wagner AD. 2013. Global similarity and pattern separation in the human medial temporal lobe predict subsequent memory. J Neurosci 33: 5466-5474.

Miller EK, Cohen JD. 2001. An integrative theory of prefrontal cortex function. Annu Rev Neurosci 24: 167-202.

Murray LJ, Ranganath C. 2007. The dorsolateral prefrontal cortex contributes to successful relational memory encoding. J Neurosci 27: 5515-5522.

Nadel L, Hoscheidt S, Ryan LR. 2013. Spatial cognition and the hippocampus: the anterior-posterior axis. J Cogn Neurosci 25: 22-28.

Navawongse R, Eichenbaum H. 2013. Distinct pathways for rule-based retrieval and spatial mapping of memory representations in hippocampal neurons. J Neurosci 33: 1002-1013.

Nee DE, Wager TD, Jonides J. 2007. Interference resolution: insights from a meta-analysis of neuroimaging tasks. Cogn Affect Behav Neurosci 7: 1-17.

Nee DE, Brown JW, Askren MK, Berman MG, Demiralp E, Krawitz A, Jonides J. 2013. A meta-analysis of executive components of working memory. Cereb Cortex 23: 264-282.

Newmark RE, Schon K, Ross RS, Stern CE. 2013. Contributions of the hippocampal subfields and entorhinal cortex to disambiguation during working memory. Hippocampus 23: 467-475.

Oren N, Shapira-Lichter I, Lerner Y, Tarrasch R, Hendler T, Giladi N, Ash EL. 2017. Schema benefit vs. proactive interference: contradicting behavioral outcomes and coexisting neural patterns. Neuroimage 158: 271-281.

Öztekin I, McElree B, Staresina BP, Davachi L. 2009. Working memory retrieval: contributions of the left prefrontal cortex, the left posterior parietal cortex, and the hippocampus. J Cogn Neurosci 21: 581-593.

Petrides M. 1994. Frontal lobes and behavior. Curr Opin Neurobiol 4: 207-211.

Petrides M. 1995. Impairments on nonspatial self-ordered and externally ordered working memory tasks after lesions of the mid-dorsal part of the lateral frontal cortex in the monkey. J Neurosci 15: 359-375.

Petrides M. 2000. Dissociable roles of mid-dorsolateral and anterior inferotemporal cortex in visual working memory. J Neurosci 20: 7496-7503.

Petrides M. 2002. The mid-ventrolateral prefrontal cortex and active mnemonic retrieval. Neurobiol Learn Mem 78: 528-538.

Petrides M. 2005. Lateral prefrontal cortex: architectonic and functional organization. Philos Trans R Soc Lond B Biol Sci 360: 781-795.

Poppenk J, Moscovitch M. 2011. A hippocampal marker of recollection memory ability among healthy young adults: contributions of posterior and anterior segments. Neuron 72: 931-937.

Poppenk J, Evensmoen HR, Moscovitch M, Nadel L. 2013. Long-axis specialization of the human hippocampus. Trends Cogn Sci 17: 230-240.

Postle BR, Berger JS, D'Esposito M. 1999. Functional neuroanatomical double dissociation of mnemonic and executive control processes contributing to working memory performance. Proc Natl Acad Sci 96: 12959-12964.

Preston AR, Eichenbaum H. 2013. Interplay of hippocampus and prefrontal cortex in memory. Curr Biol 23: R764-R773.

Psychology Software Tools, Inc. [E-Prime 2.0]. 2012. Retrieved from http:// www.pstnet.com.

Reagh ZM, Yassa MA. 2014. Object and spatial mnemonic interference differentially engage lateral and medial entorhinal cortex in humans. Proc Natl Acad Sci 111: E4264-E4273.

Rich EL, Shapiro M. 2009. Rat prefrontal cortical neurons selectively code strategy switches. J Neurosci 29: 7208-7219.

Ross RS, Brown TI, Stern CE. 2009. The retrieval of learned sequences engages the hippocampus: evidence from fMRI. Hippocampus 19: 790-799. 
Ross RS, Sherrill KR, Stern CE. 2011. The hippocampus is functionally connected to the striatum and orbitofrontal cortex during context dependent decision making. Brain Res 1423: 53-66.

Rowe JB, Toni I, Josephs O, Frackowiak RS, Passingham RE. 2000. The prefrontal cortex: response selection or maintenance within working memory? Science 288: 1656-1660.

Schendan HE, Searl MM, Melrose RJ, Stern CE. 2003. An FMRI study of the role of the medial temporal lobe in implicit and explicit sequence learning. Neuron 37: 1013-1025.

Schlichting ML, Preston AR. 2015. Memory integration: neural mechanisms and implications for behavior. Curr Opin Behav Sci 1: 1-8.

Stern CE, Owen AM, Tracey I, Look RB, Rosen BR, Petrides M. 2000. Activity in ventrolateral and mid-dorsolateral prefrontal cortex during nonspatial visual working memory processing: evidence from functional magnetic resonance imaging. Neuroimage 11: 392-399.

Stern CE, Sherman SJ, Kirchhoff BA, Hasselmo ME. 2001. Medial temporal and prefrontal contributions to working memory tasks with novel and familiar stimuli. Hippocampus 11: 337-346.
Tompary A, Davachi L. 2017. Consolidation promotes the emergence of representational overlap in the hippocampus and medial prefrontal cortex. Neuron 96: 228-241.e5.

Tzourio-Mazoyer N, Landeau B, Papathanassiou D, Crivello F, Etard O, Delcroix N, Mazoyer B, Joliot M. 2002. Automated anatomical labeling of activations in SPM using a macroscopic anatomical parcellation of the MNI MRI single-subject brain. Neuroimage 15: 273-289.

Wood ER, Dudchenko PA, Robitsek RJ, Eichenbaum H. 2000. Hippocampal neurons encode information about different types of memory episodes occurring in the same location. Neuron 27: 623-633.

Yassa MA, Stark CEL. 2011. Pattern separation in the hippocampus. Trends Neurosci 34: 515-525.

Yassa MA, Lacy JW, Stark SM, Albert MS, Gallagher M, Stark CE. 2011. Pattern separation deficits associated with increased hippocampal CA3 and dentate gyrus activity in nondemented older adults. Hippocampus 21: $968-979$.

Received November 30, 2017; accepted in revised form May 11, 2018. 


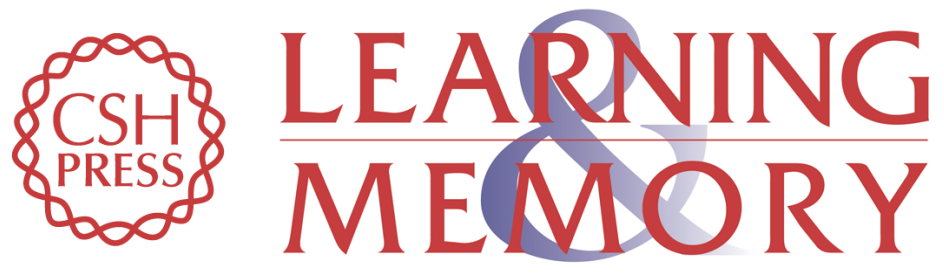

\title{
Predictability matters: role of the hippocampus and prefrontal cortex in disambiguation of overlapping sequences
}

\author{
Justine E. Cohen, Robert S. Ross and Chantal E. Stern
}

Learn. Mem. 2018, 25:

Access the most recent version at doi:10.1101/lm.047175.117

\begin{aligned} & \hline References $\begin{array}{l}\text { This article cites } 68 \text { articles, } 17 \text { of which can be accessed free at: } \\ \text { http://learnmem.cshlp.org/content/25/8/335.full.html\#ref-list-1 }\end{array} \\ & \begin{aligned} \text { Creative } \\ \text { Commons } \\ \text { License }\end{aligned} \begin{array}{l}\text { This article is distributed exclusively by Cold Spring Harbor Laboratory Press for the } \\ \text { first } 12 \text { months after the full-issue publication date (see } \\ \text { http://learnmem.cshlp.org/site/misc/terms.xhtml). After } 12 \text { months, it is available under } \\ \text { a Creative Commons License (Attribution-NonCommercial } 4.0 \text { International), as } \\ \text { described at http://creativecommons.org/licenses/by-nc/4.0/. }\end{array} \\ & \begin{array}{c}\text { Receive free email alerts when new articles cite this article - sign up in the box at the } \\ \text { top right corner of the article or click here. }\end{array} \\ & \begin{array}{c}\text { Service } \\ \text { terting }\end{array}\end{aligned}$

